\title{
Effects of 2019 novel coronavirus on male reproductive system: a retrospective study
}

Jinzhuo Ning ${ }^{*}$, Ph.D., Wei Li*2, Ph.D., Yuan Ruan* ${ }_{1}$, Ph.D., Yuqi Xia* ${ }_{1}$, MSc., Xiongfei Wu3, Ph.D., Ke Hu4, Ph.D., Xuhong Ding4, Ph.D., Xiaojun Wu4, Ph.D., Lilei Yu5, Ph.D., Jianlin Zhou6, Ph.D., Zhangfan Mao7, Ph.D., Wanzhou Xu8, Ph.D., Weimin Yu\#1, Ph.D., Fan Cheng\#1, Ph.D.

1 Department of Urology, Renmin Hospital of Wuhan University

2 Department of Anesthesiology, Renmin Hospital of Wuhan University

3 Department of Nephrology, Renmin Hospital of Wuhan University

4 Department of Respiratory Medicine, Renmin Hospital of Wuhan University

5 Department of Cardiology, Renmin Hospital of Wuhan University

6 Department of Orthopedics, Renmin Hospital of Wuhan University

7 Department of Thoracic Surgery, Renmin Hospital of Wuhan University

8 Department of Clinical Laboratory, Renmin Hospital of Wuhan University

${ }^{*}$ Contributed equally

Correspondence to: Dr Fan Cheng\# and Weimin Yu\#, Department of Urology,

Wuhan University, Renmin Hospital, Wuhan 430060, Hubei Province, P.R.

China 
E-mail: urology1969@aliyun.com (Fan Cheng) 1025931346@qq.com (Weimin Yu)

Tel.: +86-133-0710-5017; Fax: +86-027-8804-1911.

Abstract A novel and highly pathogenic coronavirus (2019-nCoV)-induced pneumonia spread worldwide in a short time. However, studies on the effects of $2019-\mathrm{nCoV}$ on the male reproductive system are limited. The aim of this study is to describe the clinical characteristics of the male reproductive system of COVID-19 patients and to explore the presence of 2019-nCoV in semen. Retrospective, single-center case series of 112 male patients with confirmed COVID-19 who were admitted to Renmin Hospital of Wuhan University from January 2 to March 7, 2020. Demographic data, symptoms and signs related to the male reproductive system, throat swabs and semen samples were collected and analyzed. 2019-nCoV RNA measured in throat swab and semen samples. The organ distribution of ACE2 mRNA and protein in human tissue on The Human Protein Atlas portal and investigated immunohistochemistry (IHC) images of the testis. The HPA dataset revealed relatively high levels of ACE2 protein and RNA expression in the testis. A total of 3 severe COVID-19 patients $(2.7 \%)$ presented with orchidoptosis, while no patients experienced other 
symptoms or signs related to the male reproductive system. The analysis of 2019-nCoV RNA in semen included 17 patients with fertility needs. Among these patients, 9 (52.9\%) remained positive for 2019-nCoV according to throat swab analysis, and $8(47.1 \%)$ became negative. In the semen 2019-nCoV analysis, all 17 patients were negative for the $\mathrm{N}$ gene and ORF1ab gene. In view of the potential impairment, long-term follow-up for male COVID-19 patients with fertility needs is of great significance.

Keywords COVID-19; 2019-nCoV; male reproductive system; a retrospective study

Since December 2019, a novel and highly pathogenic coronavirus-induced pneumonia (COVID-19) has occurred in Wuhan, Hubei Province, China1. As of 24:00 on March 17, 2020, 80894 cases were laboratory confirmed, and 3237 people had died in China according to the Chinese National Health Commission. In addition to China, the number of confirmed cases in multiple overseas countries (Italy, Germany, Iran, South Korea, etc.) continues to increase every day, which has posed a serious threat to global public health security2. Although the mortality rate of COVID-19 is not as high as that of SARS-CoV, its incidence 
rate is higher and its infectivity is stronger. Therefore, effective control of the epidemic is particularly urgent and critical.

Since the outbreak of SARS in 2003 , extensive structural analysis revealed the interaction between the spike protein receptor-binding domain (RBD) of SARS-CoV and the host cell receptor ACE2. 2019-nCoV is a kind of coronavirus highly similar to SARS-CoV; both of these viruses enter the host cell through a process mediated by their spike protein. Molecular simulation showed that the interaction between RBD and ACE2 of 2019-nCoV is stronger than that between RBD and ACE2 SARS-CoV, suggesting that ACE2 may play an important role in the process of 2019-nCoV entering host cells, which subsequently leads to infection3. A previous study showed possible testis damage in SARS patients and the effects of SARS-CoV on spermatogenesis. These findings demonstrated that SARS-CoV may cause orchitis and extensive spermatogenic cell disruption4. Fan et al.5 found that ACE2 is highly expressed in the seminiferous tubules and Leydig cells of the testis, suggesting that the testis might be a potential target of virus attack.

In the present study, we first analyzed online datasets to investigate the expression of ACE2 in testis tissue, which is the potential target of abnormal spermatogenic function in patients infected with 2019-nCoV. We retrospectively collected and analyzed 112 male patients with 2019-nCoV infection in the Renmin Hospital of Wuhan University, evaluated the clinical characteristics of the reproductive system, and preliminarily investigated the presence of 2019- 
$\mathrm{nCoV}$ in the male reproductive system by detecting 2019-nCoV in the semen of 17 patients.

\section{Methods}

\section{Study Design and Participants}

This retrospective research was approved by the ethics committee of Renmin Hospital of Wuhan University (WDRY2020-K105). Male patients over 18 years old with confirmed COVID-19 admitted to Renmin Hospital of Wuhan University from January 2 to March 7, 2020, were enrolled. The exclusion criteria for the study were a history of varicocele, orchitis, testicular trauma, testicular torsion, vasectomy, cryptorchidism or testicular biopsy that may lead to testicular alterations. No informed consent was required because the data were anonymized. Renmin Hospital, located in Wuhan, is one of the major tertiary teaching hospitals of Wuhan University and is responsible for the treatment of severe COVID-19 patients.

\section{Publicly available ACE2 expression dataset}

In this research, we used an online dataset that was approved for public research. We investigated the organ distribution of ACE2 RNA and protein expression in human tissue on The Human Protein Atlas portal (Website: http://www.proteinatlas.org/)6. Meanwhile, immunohistochemistry (IHC) images of the testis were explored. Integrated HPA datasets were sourced 
from the Genotype-Tissue Expression (GTEx) project7. All data were available online.

\section{Data Collection}

The medical records of patients were retrospectively collected and analyzed by trained physicians of the Department of Urology, Renmin Hospital of Wuhan University. Data were obtained with collection forms from electronic medical records. The information recorded included demographic data, symptoms and signs related to the male reproductive system, throat swabs and semen 2019nCoV RNA analysis. All patients with COVID-19 enrolled in this study were diagnosed and classified according to World Health Organization interim guidances. The date of disease onset was defined as the day when the symptoms were noticed. The durations from onset of disease to semen analysis were recorded.

Real-Time Reverse Transcription Polymerase Chain Reaction for 2019-nCoV

Throat swab and semen samples were collected and tested by RT-PCR for 2019-nCoV RNA. All samples were analyzed at the Department of Clinical Laboratory, Renmin Hospital of Wuhan University. Throat swab samples were collected from patients with suspected COVID-19, while semen samples were collected from confirmed male patients with fertility needs. After collection, the throat swabs and semen samples were washed or diluted with saline, and total 
RNA was extracted using an RNA extraction kit (Health Gene Technologies, Ningbo, China). Real-time RT-PCR methods targeting the open reading frame $1 \mathrm{ab}(\mathrm{ORF} 1 \mathrm{ab})$ and nucleocapsid protein $(\mathrm{N})$ genes were designed to detect 2019-nCoV RNA in patient samples. The designed primer sequences were as follows: ORF1ab, forward CCCTGTGGGTTTTACACTTAA and reverse ACGATTGTGCATCAGCTGA; the probe 5'-FAMCCGTCTGCGGTATGTGGAAAGGTTATGG-BHQ1-3'; N, forward GGGGAACTTCTCCTGCTAGAAT and reverse CAGACATTTTGCTCTCAAGCTG; and the probe 5'-FAMTTGCTGCTGCTTGA CAGATT-TAMRA-3'. The real-time RT-PCR assay was performed using a 2019-nCoV nucleic acid detection kit (Changsha, Sansure Biological Technology Co., Ltd). For all assays, the $30 \mu \mathrm{l}$ reaction mixture contained $4 \mu$ of enzyme solution and $26 \mu \mathrm{L}$ of RNA template, probe primers and reaction buffer. RT-PCR assay thermal cycling was performed at $50{ }^{\circ} \mathrm{C}$ for 30 minutes for reverse transcription, $95^{\circ} \mathrm{C}$ for 1 minute for initial denaturation, and 45 cycles of $95{ }^{\circ} \mathrm{C}$ for 15 seconds, and then $60{ }^{\circ} \mathrm{C}$ for 30 seconds for denaturation, extension and collection of the fluorescence signal. A cycle threshold value (Ct-value) less than 40 was defined as a positive test result, and a Ct-value of 40 or more was defined as a negative test. 
Categorical variables were described as frequencies and percentages, and continuous variables were described using medians, maxima and minima. All statistical analyses were performed using SPSS software version 19.0.

\section{Results}

\section{ACE2 expression level in different human tissues}

Molecular simulations showed that 2019-nCoV interacts with human host cells by binding to ACE2. To explore the potential target of 2019-nCoV, we investigated the organ distribution of ACE2 RNA and protein expression in human tissue by using online datasets. The dataset from GTEx suggested that the mRNA expression level of ACE2 is relatively higher in the small intestine and testis than in other organs (Figure $1 \mathrm{~A}$ ). Furthermore, a dataset from the HPA revealed the presence and relatively high level of ACE2 protein in the testis (Figure 1B). Meanwhile, immunohistochemistry (IHC) data from the HPA showed the presence of ACE2 protein in the testis, mainly in spermatogenic cells (Figure 1C). These results suggested that 2019-nCoV infection may cause testis injury.

\section{Presenting Characteristics}

The study population included 112 male hospitalized patients infected with 2019-nCoV. The median age was 55.5 years (range, 23-83 years). Of these patients, $40(35.7 \%)$ were classified as mild COVID-19, and $72(64.3 \%)$ were 
classified as severe COVID-19 because of the development of severe pneumonia, acute respiratory distress syndrome (ARDS) or organ dysfunction. The median age of severe patients was higher than that of mild patients. In terms of age distribution, $86 \%$ of severe patients were more than 40 years old. In the mild group, $90 \%$ of patients were less than 70 years old (Table 1 ).

\section{Symptoms and signs related to the male reproductive system}

Symptoms and signs related to the male reproductive system, including testicular pain, orchidoptosis, scrotal swelling, testicular enlargement, and testicular tenderness, were recorded on the day of hospital admission for all male patients. A total of 3 patients (2.7\%) presented with orchidoptosis for 1 to 3 days. However, no patients experienced any of the other symptoms or signs related to the male reproductive system listed above. Notably, all 3 patients had severe COVID-19, accounting for $4.2 \%$ of all severe cases (Table 1 ).

\section{Semen 2019-nCoV RNA analysis}

The study included 17 male COVID-19 patients with fertility needs whose semen was analyzed for the presence of 2019-nCoV RNA. The median age was 35 years (range, 23-46 years). The median time from onset of COVID-19 to semen detection was 27 days (range, 12-64 days). Of these patients, when we analyzed 2019-nCoV in the semen, 9 (52.9\%) remained positive for 2019$\mathrm{nCoV}$ according to the throat swab analysis, and $8(47.1 \%)$ became negative. 
In both groups, no patients experienced the symptoms or signs listed above related to the male reproductive system. Similarly, in the semen 2019-nCoV RNA analysis, all 17 patients presented negative results for the $\mathrm{N}$ gene and the ORF1ab gene (Table 2). Of these patients, 9 patients had presented severe COVID-19 but had mild COVID-19 when we performed the semen 2019-nCoV analysis.

\section{Discussion}

COVID-2019 rapidly spread throughout the country and worldwide in a very short period of time, posing a serious threat and damaging global human health. 2019-nCoV belongs to the same species as SARS-CoV, and it appears that they both use the ACE2 receptor to enter host cells for reproduction and transmission3. ACE2 is a complete membrane protein that can effectively hydrolyze vasoconstrictor II to angiotensin (1-7) and participate in the reninangiotensin system (RAS)9. It was reported that 2019-nCoV and SARS-CoV enter target host cells through the ACE2 receptor for propagation and transmission, suggesting that ACE2 can play a crucial role in the process of new coronavirus invading host cells and subsequently leading to infection 10,11. In this research, based on the organ distribution of ACE2 in human tissue revealed in online datasets, we found that the expression of ACE2 is relatively higher in the testis than in other organs and that ACE2 is mainly expressed in 
spermatogenic cells, suggesting that the testis may be potentially vulnerable to 2019-nCoV infection5,12.

In addition to typical respiratory symptoms (cough and fever), some patients may experience other symptoms, such as diarrhea, headache, fatigue, and even a few patients do not develop symptoms13. In this study, we included 112 male patients infected with $2019-\mathrm{nCoV}$ who were classified as having mild COVID-19 or severe COVID-19. A total of 3 severe patients presented with orchidoptosis for 1 to 3 days, but no patients experienced other symptoms or signs related to the male reproductive system. However, these symptoms are not specific to COVID-19. In contrast, the use semen 2019-nCoV RNA analysis might be more valuable for understanding the effects of 2019-nCoV on the male reproductive system.

The routes of transmission of 2019-nCoV include respiratory droplet transmission and contact transmission. Aerosol transmission may also occur in specific environments 14. Additionally, the virus was also detected in the feces of some patients14. The main focus of this study was to investigate the possibility of the sexual transmission of COVID-19. We analyzed the semen of 17 male patients with confirmed COVID-19 for the presence of 2019-nCoV RNA. Our results show that 2019-nCoV was not present in all of the above samples, including samples from 9 individuals that remained positive and 8 that became negative for 2019-nCoV RNA according to a throat swab analysis, suggesting that 2019-nCoV was not present in the male reproductive system of COVID-19 
patients, which is in accordance with SARS observations 15. This study is limited by the small sample size and retrospective method. Several considerations should be taken into account when interpreting the findings. First, the bloodtestis barrier is a structure located between the interstitial capillary lumen and the seminiferous tubule lumen, which consists of capillaries, endothelial cells, a basement membrane of lymphatic, myocyte-like cells, a basilar membrane of seminiferous tubules and Sertoli cells16. The blood-testis barrier can form an immune barrier. In addition to blocking the antigenicity of sperm and preventing the body from producing anti-sperm antibodies to avoid the occurrence of autoimmune reactions, it can also prevent harmful substances from interfering with spermatogenesis and damaging the formed sperm, promoting sperm production and ensuring a normal microenvironment for spermatogenesis 17. Previous studies assessed intrauterine vertical transmission by detecting the presence of 2019-nCoV in amniotic fluid and umbilical cord blood, showing no evidence of intrauterine infection in patients with a novel coronavirus during pregnancy, suggesting that the virus may not be able to transmit vertically through the placental barrier18. Second, it was also reported that Leydig cells and testicular macrophages have strong antiviral ability, which can produce interferon $\alpha$ and $y$ simultaneously after contact with viruses, thereby resisting viruses and tumors and enhancing immune regulatory function 19,20. Meanwhile, studies have shown that Leydig cells and macrophages secrete a variety of antiviral proteases under the stimulation of Sendai virus, which indicated that 
in addition to the blood-testis barrier, the testicular system also an ideal antiviral system21.. Third, in view of the potential impairment of spermatogenic function by $2019-n C o V$ infection, and the current observation and follow-up of 2019$\mathrm{nCoV}$ patients is limited to the hospitalization and recovery period; the time is relatively short.

For male patients with fertility needs, the review and long-term follow-up of male spermatogenic function are of great significance. Color Doppler ultrasound and routine semen analysis are frequently used imaging methods and male fertility testing methods suitable for male 2019-nCoV patients with scrotal discomfort, testicular swelling and pain, or fertility needs. Sex hormone tests can assess gonadal endocrine dysfunction, and they are suitable for 2019-nCoV patients with abnormal semen analysis results. Studies have shown that testosterone and luteinizing hormone are closely related to sperm count and sperm motility22. Viral orchitis may cause testicular mesenchymal cell damage, decreased testosterone levels and increased gonadotropin levels, leading to secondary hypogonadism23. 2019-nCoV patients with infertility or abnormal semen analysis results should be tested for sperm acrosome function, sperm nuclear DNA fragments, and antisperm antibodies to evaluate the intrinsic causes of abnormal sperm function.

In summary, front-line medical staff need to pay close attention to the testicular function of 2019-nCoV patients, especially for patients with future fertility requirements. Not only to pay attention to changes in the local symptoms 
and signs of the testis during the course of the disease but also to perform longterm follow-up of testicular spermatogenesis functional examination.

\section{Conclusions}

The online datasets indicated the potential impairment of the male reproductive system by 2019-nCoV. However, this group of cases suggested that male patients have few reproductive symptoms and signs, and 2019-nCoV was not present in the male reproductive system of patients with confirmed COVID-19. In view of the potential impairment, the long-term follow-up for male COVID-19 patients with fertility needs is of great significance.

\section{Acknowledgement}

This research was supported by grants from the Science and Technology Major Project of Hubei Province (No. 2019AEA170), and we would like to especially thank the doctor teams from all over the country, for their full support to Wuhan, Hubei.

\section{Conflicts of interest}

All authors declare no conflicts of interest.

\section{REFERENCES}

1. Lu H, Stratton CW, Tang YW. Outbreak of pneumonia of unknown etiology in Wuhan, China: The mystery and the miracle. Journal of medical virology. 2020;92(4):401-402.

2. Bogoch, II, Watts A, Thomas-Bachli A, et al. Potential for global spread of a novel coronavirus 
from China. Journal of travel medicine. 2020;27(2).

3. Chen Y, Guo Y, Pan Y, et al. Structure analysis of the receptor binding of 2019-nCoV. Biochemical and biophysical research communications. 2020.

4. Xu J, Qi L, Chi X, et al. Orchitis: a complication of severe acute respiratory syndrome (SARS). Biology of reproduction. 2006;74(2):410-416.

5. Fan C, Li K, Ding Y, et al. ACE2 Expression in Kidney and Testis May Cause Kidney and Testis Damage After 2019-nCoV Infection. medRxiv. 2020:2020.2002.2012.20022418.

6. Uhlen M, Fagerberg L, Hallstrom BM, et al. Proteomics. Tissue-based map of the human proteome. Science (New York, NY). 2015;347(6220):1260419.

7. Barretina J, Caponigro G, Stransky N, et al. The Cancer Cell Line Encyclopedia enables predictive modelling of anticancer drug sensitivity. Nature. 2012;483(7391):603-607.

8. WHO. Clinical management of severe acute respiratory infection when novel coronavirus (nCoV) infection is suspected. Interim guidance. https://www.who.int/docs/defaultsource/coronaviruse/clinical-management-of-novel-cov. 2020.

9. Watermeyer JM, Sewell BT, Schwager SL, et al. Structure of testis ACE glycosylation mutants and evidence for conserved domain movement. Biochemistry. 2006;45(42):12654-12663.

10. Zhou P, Yang XL, Wang XG, et al. A pneumonia outbreak associated with a new coronavirus of probable bat origin. Nature. 2020;579(7798):270-273.

11. Li W, Moore MJ, Vasilieva N, et al. Angiotensin-converting enzyme 2 is a functional receptor for the SARS coronavirus. Nature. 2003;426(6965):450-454.

12. Wang Z XX. scRNA-seq Profiling of Human Testes Reveals the Presence of ACE2 Receptor, a Target for SARS-CoV-2 Infection, in Spermatogonia, Leydig and Sertoli Cells. Preprints 2020. 2020;2020020299.

13. Wang D, Hu B, Hu C, et al. Clinical characteristics of 138 hospitalized patients with 2019 novel coronavirus-infected pneumonia in Wuhan, China. Jama. 2020.

14. Young BE, Ong SWX, Kalimuddin S, et al. Epidemiologic features and clinical course of patients infected with SARS-CoV-2 in Singapore. JAMA. 2020.

15. Ding $\mathrm{Y}, \mathrm{He} \mathrm{L}$, Zhang $\mathrm{Q}$, et al. Organ distribution of severe acute respiratory syndrome (SARS) associated coronavirus (SARS-CoV) in SARS patients: implications for pathogenesis and virus transmission pathways. The Journal of pathology. 2004;203(2):622-630.

16. Mruk DD, Cheng CY. The Mammalian Blood-Testis Barrier: Its Biology and Regulation. Endocrine reviews. 2015;36(5):564-591.

17. Cheng CY, Mruk DD. The blood-testis barrier and its implications for male contraception. 
Pharmacological reviews. 2012;64(1):16-64.

18. Chen H, Guo J, Wang C, et al. Clinical characteristics and intrauterine vertical transmission potential of COVID-19 infection in nine pregnant women: a retrospective review of medical records. Lancet (London, England). 2020;395(10226):809-815.

19. Dejucq N, Dugast I, Ruffault A, van der Meide PH, Jegou B. Interferon-alpha and -gamma expression in the rat testis. Endocrinology. 1995;136(11):4925-4931.

20. Scott DW. Immunophysiology: The role of cells and cytokines in immunity and inflammation. Pathology. 1990;4(3):265-265.

21. Melaine N, Lienard MO, Guillaume E, et al. Production of the antiviral proteins 2'5'oligoadenylate synthetase, PKR and Mx in interstitial cells and spermatogonia. Journal of reproductive immunology. 2003;59(1):53-60.

22. Ring JD, Lwin AA, Kohler TS. Current medical management of endocrine-related male infertility. Asian journal of andrology. 2016;18(3):357-363.

23. $\mathrm{Ku} \mathrm{JH}, \mathrm{Kim} \mathrm{YH}$, Jeon YS, et al. The preventive effect of systemic treatment with interferonalpha2B for infertility from mumps orchitis. BJU international. 1999;84(7):839-842. 
Figure legends
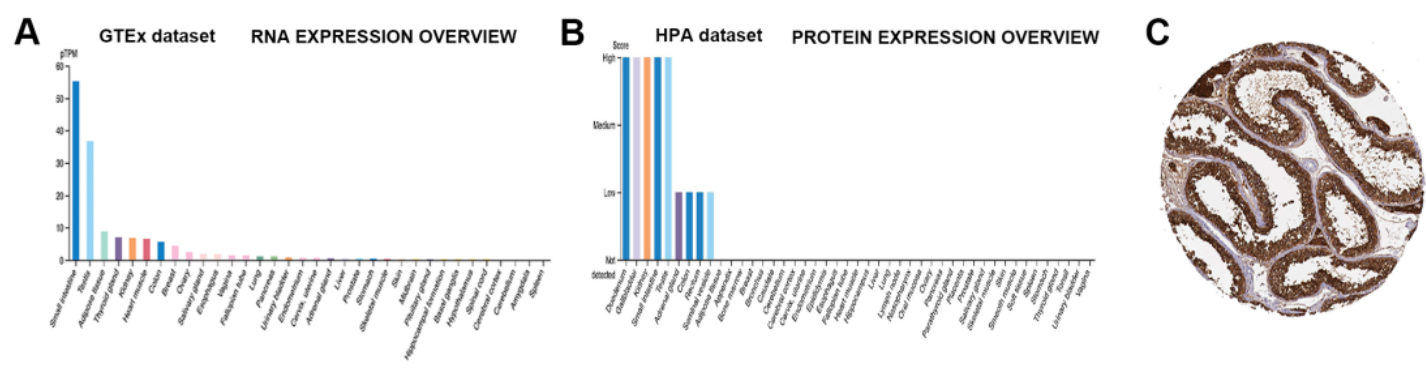

Figure1. Data of ACE2 from GTEx and HPA dataset. A, B. Data of ACE2 RNA and protein expression level in different human tissues from GTEx and HPA dataset. C. Representative IHC staining of ACE2 in testis

Table1. Clinical Features of Patients Infected with 2019-nCoV

$\begin{array}{ccc}\text { All patients } & \text { Mild group } & \text { Severe group } \\ (\mathrm{N}=112) & (\mathrm{N}=40) & (\mathrm{N}=72)\end{array}$

\begin{tabular}{lccc}
\hline Age, years & & & \\
\hline Median(range) & $55.5(23-83)$ & $45.5(24-82)$ & $57(23-83)$ \\
\hline$\leq 39$ & $25(22 \%)$ & $15(38 \%)$ & $10(14 \%)$ \\
\hline $40-54$ & $29(26 \%)$ & $10(25 \%)$ & $19(26 \%)$ \\
\hline $55-69$ & $43(39 \%)$ & $11(27 \%)$ & $32(45 \%)$ \\
\hline$\geq 70$ & $15(14 \%)$ & $4(10 \%)$ & $11(15 \%)$ \\
\hline $\begin{array}{l}\text { Symptoms on male } \\
\text { reproductive system, No }\end{array}$ & & & \\
\hline \multicolumn{1}{l}{ Testicular pain } & 0 & 0 & 0 \\
$\quad$ Orchidoptosis & $3(2.7 \%)$ & 0 & $3(4.2 \%)$ \\
\hline $\begin{array}{l}\text { Signs on male reproductive } \\
\text { system, No }\end{array}$ & & & \\
\hline Scrotal swelling & 0 & 0 & 0 \\
\hline Testicular enlargement & 0 & 0 & 0 \\
\hline Testicular tenderness & 0 & 0 & 0 \\
\hline
\end{tabular}


Abbreviations: 2019-nCoV, 2019-novel Coronavirus. N, Nucleoprotein gene. ORF, Open reading frame.

Table2. Clinical Features of Patients Detected Semen 2019-nCoV RNA

\begin{tabular}{|c|c|c|c|}
\hline & $\begin{array}{l}\text { All patients } \\
\qquad(\mathrm{N}=17)\end{array}$ & $\begin{array}{c}\text { Throat swab } \\
\text { remained positive } \\
(\mathrm{N}=9)\end{array}$ & $\begin{array}{c}\text { Throat swab } \\
\text { converted into } \\
\text { negative }(\mathrm{N}=8)\end{array}$ \\
\hline \multicolumn{4}{|l|}{ Age, years } \\
\hline Median(range) & $35(23-46)$ & $38(23-46)$ & $30(28-45)$ \\
\hline$\leq 39$ & $13(76 \%)$ & $7(78 \%)$ & $6(75 \%)$ \\
\hline $40-54$ & $4(24 \%)$ & $2(22 \%)$ & $2(25 \%)$ \\
\hline$\geq 55$ & 0 & 0 & 0 \\
\hline $\begin{array}{l}\text { Onset of COVID-19 to semen } \\
\text { detection, median(range) }\end{array}$ & $27(12-64)$ & $30(14-64)$ & $18.5(12-36)$ \\
\hline \multicolumn{4}{|l|}{$\begin{array}{l}\text { Symptoms on male reproductive } \\
\text { system, No }\end{array}$} \\
\hline Scrotum discomfort & 0 & 0 & 0 \\
\hline Testicular pain & 0 & 0 & 0 \\
\hline Orchidoptosis & 0 & 0 & 0 \\
\hline \multicolumn{4}{|l|}{$\begin{array}{l}\text { Signs on male reproductive } \\
\text { system, No }\end{array}$} \\
\hline Scrotal swelling & 0 & 0 & 0 \\
\hline Testicular enlargement & 0 & 0 & 0 \\
\hline Testicular tenderness & 0 & 0 & 0 \\
\hline \multicolumn{4}{|l|}{ Semen Detection of 2019-nCoV } \\
\hline \multicolumn{4}{|l|}{ RNA } \\
\hline 2019-nCOV NP positive & 0 & 0 & 0 \\
\hline 2019-nCOV ORF1ab positive & 0 & 0 & 0 \\
\hline
\end{tabular}


Abbreviations: COVID-19, corona virus infective disease 2019. 2019-nCoV, 2019-novel Coronavirus. N, Nucleoprotein gene. ORF, Open reading frame. 